\title{
A note on permanence of nonautonomous cooperative scalar population models with delays
}

\author{
Teresa Faria ${ }^{1}$ \\ Departamento de Matemática and CMAF \\ Faculdade de Ciências, Universidade de Lisboa, \\ Campo Grande, 1749-016 Lisboa, Portugal \\ tfaria@ptmat.fc.ul.pt
}

\begin{abstract}
For a large family of nonautonomous scalar-delayed differential equations used in population dynamics, some criteria for permanence are given, as well as explicit upper and lower bounds for the asymptotic behavior of solutions. The method described here is based on comparative results with auxiliary monotone systems. In particular, it applies to a nonautonomous scalar model proposed as an alternative to the usual delayed logistic equation.
\end{abstract}

Keywords: Delay differential equation; permanence; cooperative equation; quasimonotone condition. 2010 Mathematics Subject Classification: 34K12, 34K25, 92D25

\section{Introduction}

In [3], Bastinec et al. studied the permanence of the following scalar nonautonomous delay differential equation (DDE) with a quadratic nonlinearity:

$$
\dot{x}(t)=\sum_{k=1}^{m} \alpha_{k}(t) x\left(t-\tau_{k}(t)\right)-\beta(t) x^{2}(t), \quad t \geq 0
$$

where $m$ is a positive integer, $\alpha_{k}, \beta:[0, \infty) \rightarrow(0, \infty)$ are continuous, $\tau_{k}:[0, \infty) \rightarrow[0, \infty)$ are continuous and uniformly bounded, $0 \leq \tau_{k}(t) \leq \tau$ for some $\tau>0$, for $k=1, \ldots, m, t \geq 0$.

In view of the biological interpretation of model (1.1), only positive (or nonnegative) solutions of (1.1) are meaningful. In [3], the authors restrict their attention to solutions of (1.1) with initial conditions of the form

$$
x(\theta)=\varphi(\theta),-\tau \leq \theta \leq 0,
$$

for $\varphi:[0, \infty) \rightarrow(0, \infty)$ continuous, and added the contraint $\sum_{k=1}^{m} \tau_{k}(t)>0$ for all $t \geq 0$. Using the positivity of the functions $\alpha_{k}(t), \beta(t)$, it is easy to see that solutions of (1.1)-(1.2) are positive whenever they are defined.

In a previous paper [2], the same authors considered a simpler nonautonomous model

$$
\dot{x}(t)=r(t)\left[\sum_{k=1}^{m} \alpha_{k} x\left(t-\tau_{k}(t)\right)-\beta x^{2}(t)\right], \quad t \geq 0,
$$

\footnotetext{
${ }^{1}$ Fax:+351 21795 4288; Tel: +35121790 4929.
} 
where the delay functions $\tau_{k}(t)$ satisfy all the conditions above, $r(t)$ is continuous and satisfies $r(t) \geq$ $r_{0}, t \geq 0$, for some constant $r_{0}>0$, and $\alpha_{k}, \beta$ are positive constants, $1 \leq k \leq m$.

Model (1.1) is a generalization of the DDE (1.3), obtained by considering a more general form of nonautonomous coefficients. The scalar DDE (1.3) has a positive equilibrium $K^{*}=\frac{1}{\beta} \sum_{k=1}^{m} \alpha_{k}$, which was proven in 2] to be a global attractor of all its positive solutions without any further restriction. In general, (1.1) does not have a positive equilibrium, so criteria for either extinction - when zero is a global attractor - or persistence or permanence play a crucial role.

Here, we set some standard notations. For (1.1) and for the DDEs hereafter, $C:=C([-\tau, 0] ; \mathbb{R})$ $(\tau>0)$ with the usual sup norm $\|\varphi\|_{\infty}=\sup _{\theta \in[-\tau, 0]}|\varphi(\theta)|$ will be taken as the phase space. For an abstract DDE in $C$,

$$
\dot{x}(t)=f\left(t, x_{t}\right), \quad t \geq t_{0},
$$

where $f: \Omega \subset \mathbb{R} \times C \rightarrow \mathbb{R}$ is continuous, $x_{t}$ denotes segments of solutions in $C, x_{t}(\theta)=x(t+\theta),-\tau \leq$ $\theta \leq 0$. If the solutions of initial value problems are unique, $x\left(t ; t_{0}, \varphi\right)$ designates the solution of $\dot{x}(t)=f\left(t, x_{t}\right), x_{t_{0}}=\varphi$; we use simply $x(t ; \varphi)$ for $x(t ; 0, \varphi)$. Even if it is not stated, we shall always assume that $f$ is smooth enough so that initial value problems associated with (1.4) have unique solutions, with continuous dependence on data. This is the case if $f(t, \varphi)$ is uniformly Lipschitz continuous on the variable $\varphi \in C$ on each compact subset of $\Omega$. For $C^{+}:=\{\varphi \in C: \varphi(\theta) \geq 0$ for $-\tau \leq \theta \leq 0\}$, initial conditions (1.2) are written in the simpler form $x_{0}=\varphi$ with $\varphi \in \operatorname{int}\left(C^{+}\right)$. Cf. e.g. [5], for the concept of permanence given below, as well as for other standard definitions.

Definition 1.1. The scalar DDE (1.4) is said to be permanent (in $S=\operatorname{int}\left(C^{+}\right)$, or another $S \subset$ $\left.C^{+} \backslash\{0\}\right)$ if there are positive constants $m_{0}, M_{0}$ with $m_{0}<M_{0}$ such that, given any $\varphi \in S$, there exists $t_{*}=t_{*}(\varphi)$ such that $m_{0} \leq x(t, \varphi) \leq M_{0}$ for $t \geq t_{*}$.

A nice criterion for the permanence of (1.1) was established in [3], assuming only that the functions $\alpha_{k}(t), \beta(t)$ are uniformly bounded from above and from below by positive constants.

Theorem 1.1. [3] Assume that $\sum_{k=1}^{m} \tau_{k}(t)>0$ for all $t \geq 0$. If

(h1) there are positive constants $\alpha_{0}, A_{0}, \beta_{0}, B_{0}$ such that

$$
\alpha_{0} \leq \alpha_{k}(t) \leq A_{0}, \quad \beta_{0} \leq \beta(t) \leq B_{0} \quad \text { for } \quad t \geq 0, k=1, \ldots, m,
$$

then the solutions of the initial value problems (1.1)-(1.2) are positive and defined on $[0, \infty)$, and (1.1) is permanent in int $\left(C^{+}\right)$. Moreover, for every solution $x(t)$ of (1.1) -(1.2) the estimates

$$
m_{0} \leq \liminf _{t \rightarrow \infty} x(t) \leq \limsup _{t \rightarrow \infty} x(t) \leq M_{0},
$$

hold with

$$
m_{0}=\liminf _{t \rightarrow \infty} \frac{1}{\beta(t)} \sum_{k=1}^{m} \alpha_{k}(t), \quad M_{0}=\limsup _{t \rightarrow \infty} \frac{1}{\beta(t)} \sum_{k=1}^{m} \alpha_{k}(t) .
$$

The proof of this result in [3] is broken into several steps, and takes little advantage of the criterion established previously by the authors in [2]. Here, we present an alternative proof based on the fact that equations (1.1) and (1.3) satisfy the quasimonotone condition. In fact, we shall show later (cf. Theorem 3.2) that we need not assume that $\sum_{k=1}^{m} \tau_{k}(t)>0$ for all $t \geq 0$, and that initial conditions may be taken in the larger set $C_{0}:=\left\{\varphi \in C^{+}: \varphi(0)>0\right\}$. We recall that a scalar DDE (1.4) satisfies the quasimonotone condition (on the cone $C^{+}$) if for any $t \geq t_{0}$ and $\varphi, \psi \in C^{+}$with $\varphi \leq \psi$ and $\varphi(0)=\psi(0)$, then $f(t, \varphi) \leq f(t, \psi)$ (cf. [7], p. 78). Under this condition, the semiflow is monotone. If $d_{\varphi} f(t, \varphi)$ exists, is continuous on $\left[t_{0}, \infty\right) \times C^{+}$, and $d_{\varphi} f(t, \varphi) \psi \geq 0$ for $\varphi, \psi \in C^{+}$ and $\psi(0)=0$, then (1.4) is cooperative; cooperative equations satisfy the quasimonotone condition. Here, we abuse the terminology, and refer to equations satisfying the quasimonotone condition as cooperative. 
Alternative proof of Theorem 1.1. Let $x(t)=x(t ; \varphi)$ be the solution for an initial value problem (1.1)(1.2), defined on some maximal interval $[0, a)$ with $a \in(0, \infty]$. Then $x(t)$ satisfies the inequality $\dot{x}(t) \leq A_{0} \sum_{k=1}^{m} x\left(t-\tau_{k}(t)\right)-\beta_{0} x^{2}(t), t \geq 0$. Comparing with the cooperative equation

$$
\dot{u}(t)=A_{0} \sum_{k=1}^{m} u\left(t-\tau_{k}(t)\right)-\beta_{0} u^{2}(t), \quad t \geq 0,
$$

by Theorem 5.1.1 of 7] we have that $x(t)$ is bounded and defined on $[0, \infty)$, with $x(t) \leq u(t), t \geq 0$, where $u(t)$ is the solution of (1.7)-(1.2). Moreover, by [2] the equilibrium $u^{*}=\frac{m A_{0}^{-}}{\beta_{0}}$ is a global attractor for all positive solutions of (1.7). We therefore conclude that $\lim _{\sup } \operatorname{sum}_{t \rightarrow \infty} x(t) \leq u^{*}$. In a similar way, we have $\dot{x}(t) \geq \alpha_{0} \sum_{k=1}^{m} x\left(t-\tau_{k}(t)\right)-B_{0} x^{2}(t), t \geq 0$, and by comparison with the cooperative equation

$$
\dot{v}(t)=\alpha_{0} \sum_{k=1}^{m} v\left(t-\tau_{k}(t)\right)-B_{0} v^{2}(t), \quad t \geq 0,
$$

we obtain the lower bound $\liminf \operatorname{in}_{t \rightarrow \infty} x(t) \geq v^{*}:=\frac{m \alpha_{0}}{B_{0}}$. Hence, (1.1) is permanent.

We now prove the estimates in (1.5)-(1.6). Denote $\underline{x}=\liminf _{t \rightarrow \infty} x(t), \bar{x}=\limsup _{t \rightarrow \infty} x(t)$. By the fluctuation lemma, there is a sequence $\left(t_{n}\right)$, with $t_{n} \rightarrow \infty$ and $x\left(t_{n}\right) \rightarrow \bar{x}, \dot{x}\left(t_{n}\right) \rightarrow 0$. Fix a small $\varepsilon>0$, and take $T>0$ so that $x(t) \leq \bar{x}+\varepsilon$ for $t \geq T+\tau$. For $n$ large enough so that $t_{n}-\tau_{k}\left(t_{n}\right) \geq T$ and $x\left(t_{n}\right) \geq \bar{x}-\varepsilon$, using (1.1) we get

$$
\begin{aligned}
\dot{x}\left(t_{n}\right) & =\beta\left(t_{n}\right)\left[\sum_{k=1}^{m} \frac{\alpha_{k}\left(t_{n}\right)}{\beta\left(t_{n}\right)} x\left(t_{n}-\tau_{k}\left(t_{n}\right)\right)-x^{2}\left(t_{n}\right)\right] \\
& \leq \beta\left(t_{n}\right)\left[\frac{\bar{x}+\varepsilon}{\beta\left(t_{n}\right)} \sum_{k=1}^{m} \alpha_{k}\left(t_{n}\right)-(\bar{x}-\varepsilon)^{2}\right] \\
& \leq \beta\left(t_{n}\right) \bar{x}\left[\frac{1}{\beta\left(t_{n}\right)} \sum_{k=1}^{m} \alpha_{k}\left(t_{n}\right)-\bar{x}\right]+O(\varepsilon) .
\end{aligned}
$$

By letting $n \rightarrow \infty$ and $\varepsilon \rightarrow 0^{+}$, we obtain

$$
0 \leq \limsup _{n} \beta\left(t_{n}\right)\left[\frac{1}{\beta\left(t_{n}\right)} \sum_{k=1}^{m} \alpha_{k}\left(t_{n}\right)-\bar{x}\right],
$$

which implies $M_{0}-\bar{x} \geq 0$, for $M_{0}$ as in (1.6). The other inequality in (1.5) is proven in a similar way.

The above method used to prove Theorem 1.1 motivated us to extend the same arguments to other scalar DDEs from population dynamics. The idea is to consider a broad class of cooperative differential equations with (possibly time-varying) delays and nonautonomous coefficients, and use the theory of monotone dynamical systems to obtain its permanence by comparison with two auxiliary differential equations with constant coefficients, for which a globally attractive positive equilibrium exists.

As a particularly important example, we have in mind to apply this approach to establish the permanence of the following scalar population model:

$$
\dot{x}(t)=\sum_{k=1}^{m} \frac{\alpha_{k}(t) x\left(t-\tau_{k}(t)\right)}{1+\beta_{k}(t) x\left(t-\tau_{k}(t)\right)}-\mu(t) x(t)-\kappa(t) x^{2}(t), \quad t \geq 0,
$$

where $\alpha_{k}, \kappa:[0, \infty) \rightarrow(0, \infty), \beta_{k}, \mu, \tau_{k}:[0, \infty) \rightarrow[0, \infty)$ are continuous and bounded, $1 \leq k \leq m$. We emphasize that the study of the permanence of (1.8) (with the additional constraints $\beta_{k}(t)>0, \mu(t)>$ 
$0, \sum_{k=1}^{m} \tau_{k}(t)>0$ on $\left.[0, \infty)\right)$ was proposed in [3] as a topic for further research. Obviously, Theorem 1.1 applies only to the very concrete model (1.1), and therefore cannot be invoked to deal with (1.8). For $N(t)=x(t)$, eq. (1.8) with $m=1$ reads as

$$
N^{\prime}(t)=\frac{\alpha(t) N(t-\tau(t))}{1+\beta(t) N(t-\tau(t))}-\mu(t) N(t)-\kappa(t) N^{2}(t),
$$

which is (after a scaling) the nonautonomous version of

$$
N^{\prime}(t)=\frac{\gamma \mu N(t-\tau)}{\mu e^{\mu \tau}+k\left(e^{\mu \tau}-1\right) N(t-\tau)}-\mu N(t)-\kappa N^{2}(t),
$$

where $\gamma, \mu, \kappa, \tau>0$. Eq. (1.9) was derived by Arino et al. [1] as an alternative formulation for the classical delayed logistic equation, also known as Wright's equation, given by $\dot{N}(t)=r N(t)(1-N(t-$ $\tau) / K)$, where $r$ denotes the intrinsic growth rate, $K$ is the carrying capacity, and $\tau$ the maturation delay. The coefficients in this logistic equation are related to the ones in (1.9) by $r=\gamma-\mu$ (for $\gamma, \mu$ the birth and mortality rates, respectively) and $K=(\gamma-\mu) / \kappa$. In Section 3 , we shall study a class of scalar DDEs which includes (1.8) as a particular case. As another illustration of our technique, the permanence of a nonautonomous Nicholson's blowflies equation will also be studied.

\section{Auxiliary results on stability of equilibria}

In this section, we address the global attractivity of nonnegative equilibria for a family of nonautonomous cooperative scalar DDEs. We start with an auxiliary lemma from [4].

Lemma 2.1. [4] Consider a scalar equation (1.4) in $C=C([-\tau, 0] ; \mathbb{R})$, with $f:\left[t_{0}, \infty\right) \times C \rightarrow \mathbb{R}$ continuous, $t_{0} \in \mathbb{R}$, and let $S \subset C$ be an invariant set for the semiflow of (1.4). Suppose that $f$ satisfies the condition

(h2) for $t \geq t_{0}$ and $\varphi \in S$ with $|\varphi(\theta)|<|\varphi(0)|, \theta \in[-\tau, 0)$, then $\varphi(0) f(t, \varphi)<0$.

Then, the solutions of (1.4) with initial conditions $x_{0}=\varphi \in S$ are defined and bounded on $\left[t_{0}, \infty\right)$ and $|x(t)| \leq\|\varphi\|_{\infty}$ for $t \geq t_{0}$.

Consider now the family of scalar-delayed population models given by

$$
\dot{x}(t)=\rho(t)\left[R\left(x\left(t-\tau_{1}(t)\right), \ldots, x\left(t-\tau_{m}(t)\right)\right)-D(x(t))\right], \quad t \geq 0,
$$

where: $m \in \mathbb{N}, \tau_{k}:[0, \infty) \rightarrow \mathbb{R}$ are continuous and $0 \leq \tau_{k}(t) \leq \tau$ for some positive constant $\tau$, for $t \geq 0,1 \leq k \leq m ; \rho:[0, \infty) \rightarrow(0, \infty), R: \mathbb{R}_{+}^{m}:=[0, \infty)^{m} \rightarrow[0, \infty), D:[0, \infty) \rightarrow[0, \infty)$ are continuous with $R(0, \ldots, 0)=D(0)=0$. Moreover, let $R, D$ be smooth enough in order to ensure uniqueness of solutions. The condition $R(0, \ldots, 0)=0$ is not essential for our analysis, but it corresponds to the general framework in population dynamics models, since zero should be a steady state. We note that the particular case $\dot{x}(t)=R(x(t-\tau))-D(x(t)), t \geq 0$, was studied by Arino et al [1].

Write (2.1) as $\dot{x}(t)=f\left(t, x_{t}\right)$, and observe that $f$ satisfies Smith's quasimonotone condition. As before, due to biological reasons we are only interested in positive solutions. Rather than initial conditions in $\operatorname{int}\left(C^{+}\right)$, we shall consider $x_{0}=\varphi$, with $\varphi$ in the larger set of admissible initial conditions

$$
C_{0}=\left\{\varphi \in C^{+}: \varphi(0)>0\right\} .
$$

It is clear that for $t \geq 0$ and $\varphi \in C^{+}$with $\varphi(0)=0$, then $f(t, \varphi) \geq 0$. This implies that solutions of (2.1) with initial conditions $x_{0} \in C^{+}$are nonnegative [7]. For $x(t)=x(t ; \varphi), \varphi \in C_{0}, x(t)$ satisfies the ordinary differential inequality $\dot{x}(t) \geq-\rho(t) D(x(t))$, thus conditions $D(0)=0$ and $x(0)=\varphi(0)>0$ yield $x(t)>0$ whenever it is defined. In what follows, concepts as permanence and global asymptotic stability always refer to the solutions with initial conditions $x_{0}=\varphi \in C_{0}$.

In the sequel, the following assumptions will be considered: 
(A1) $\rho(t)$ is bounded from below by a positive constant, i.e., $\rho(t) \geq \rho_{0}>0$ for all $t \geq 0$;

(A2) $R\left(y_{1}, \ldots, y_{m}\right)$ is nondecreasing in $y_{k} \in[0, \infty), k=1, \ldots, m$;

(A3) there exists $K \geq 0$ such that

$$
(x-K)(R(x, \ldots, x)-D(x))<0 \quad \text { for } \quad x>0, x \neq K .
$$

Note that if there exists $K>0$ such that (2.2) holds, then (2.1) has the equilibria 0 and $K$; whereas 0 is the unique equilibrium if (2.2) is satisfied with $K=0$.

Next result is a generalization of Theorem 3.3 in [1], and its proof can be found in the Appendix. For related results, see [4] and Chapter 4 of Kuang's monograph [5].

Theorem 2.1. Consider equation (2.1), and assume (A1)-(A3). Then the equilibrium $K$ in (A3) is globally asymptotically stable $(G A S)$ in the set of solutions with initial conditions in $C_{0}$.

The same proof works with minimal changes for equations with distributed delays, rather than discrete delays, as stated below.

Theorem 2.2. Consider equation

$$
\dot{x}(t)=\rho(t)\left[R\left(L_{1}\left(t, x_{t}\right), \ldots, L_{m}\left(t, x_{t}\right)\right)-D(x(t))\right], \quad t \geq 0,
$$

where $R, D$ are as in (2.1), and $L_{k}:[0, \infty) \times C \rightarrow \mathbb{R}$ are defined by

$$
L_{k}(t, \varphi)=\int_{-\tau}^{0} \varphi(\theta) d_{\theta} \eta_{k}(t, \theta), \quad t \geq 0, \varphi \in C,
$$

for some measurable functions $\eta_{k}:[0, \infty) \times[-\tau, 0] \rightarrow \mathbb{R}$ such that $\eta_{k}(t, \cdot)$ is nondecreasing on $[-\tau, 0]$ with $\eta_{k}(t, 0)-\eta_{k}(t,-\tau)=1$ for all $t \geq 0, k=1, \ldots, m$. If (A1)-(A3) are satisfied, then the equilibrium $K$ in (A3) is GAS in the set of solutions with initial conditions in $C_{0}$.

For $L_{k}$ as in the above statement, $L_{k}(t, \cdot): C \rightarrow \mathbb{R}$ are normalized, positive linear operators for all $t \geq 0$. Clearly, eq. (2.1) is a particular case of (2.3), where $\eta_{k}(t, \theta)$ are Heaviside functions, $\eta_{k}(t, \theta)=0$ for $-\tau \leq \theta \leq-\tau_{k}(t), \eta_{k}(t, \theta)=1$ for $-\tau_{k}(t)<\theta \leq 0$.

We now consider a subclass of scalar DDEs (2.1), since a significant number of population models have the form (2.1) (or (2.3)) with $R\left(y_{1}, \ldots, y_{m}\right)=\sum_{k=1}^{m} y_{k} r_{k}\left(y_{k}\right), D(x)=x d(x)$ for $\left(y_{1}, \ldots, y_{m}\right) \in$ $\mathbb{R}_{+}^{m}, x \in \mathbb{R}_{+}$, with $r_{k}, d$ strictly positive on $\mathbb{R}_{+}$. See Theorem 3.3 in [1] for a particular case, and [ 5 , pp. 146], also for further references.

Corollary 2.1. Consider the equation

$$
\dot{x}(t)=\sum_{k=1}^{m} x\left(t-\tau_{k}(t)\right) r_{k}\left(x\left(t-\tau_{k}(t)\right)\right)-x(t) d(x(t)), \quad t \geq 0,
$$

where $r_{k}, d:[0, \infty) \rightarrow[0, \infty)$ are locally Lipschitz functions with $r_{k}(x)>0, d(x)>0$ for $x>0$, and $\tau_{k}:[0, \infty) \rightarrow[0, \tau]$ are continuous, $k=1, \ldots, m$. Assume also that

(i) $x r_{k}(x)$ are nondecreasing functions on $[0, \infty), k=1, \ldots, m$;

(ii) for $r(x):=\sum_{k=1}^{m} r_{k}(x)$, the function $r(x)-d(x)$ is (strictly) decreasing on $[0, \infty)$;

(iii) $r(\infty)-d(\infty)<0$. 
Then, there are at most two nonnegative equilibria. If zero is the unique equilibrium, then it is GAS; if there is a positive equilibrium $x^{*}$, then $x^{*}$ is GAS (in the set of all solutions with initial conditions $\left.x_{0}=\varphi \in C_{0}\right)$.

Proof. If $r(0)-d(0) \leq 0$, (A3) holds with $K=0$, hence 0 is GAS; if $r(0)-d(0)>0$, then there exists a unique $x^{*}>0$ such that $r\left(x^{*}\right)-d\left(x^{*}\right)=0$, and (A3) holds with $K=x^{*}$.

A similar version of this corollary for equations with distributed delays could also be stated.

\section{Main results}

Based on Theorem 2.1, we now extend the arguments used in our proof of Theorem 1.1 to a larger class of nonautonomous cooperative models.

Theorem 3.1. Consider

$$
\dot{x}(t)=R\left(t, x\left(t-\tau_{1}(t)\right), \ldots, x\left(t-\tau_{m}(t)\right)\right)-D(t, x(t)), \quad t \geq 0,
$$

where $m \in \mathbb{N}, R(t, y), D(t, x), \tau_{k}(t)$ are continuous with $0 \leq \tau_{k}(t) \leq \tau$, for $t, x \in \mathbb{R}_{+}, y \in \mathbb{R}_{+}^{m}, 1 \leq k \leq$ $m$, and assume that:

(H1) there are (locally Lipschitz) continuous functions $R^{l}, R^{u}: \mathbb{R}_{+}^{m} \rightarrow \mathbb{R}_{+}, D^{l}, D^{u}: \mathbb{R}_{+} \rightarrow \mathbb{R}_{+}$with $R^{l}(0, \ldots, 0)=R^{u}(0, \ldots, 0)=D^{l}(0)=D^{u}(0)=0$, such that

$$
R^{l}(y) \leq R(t, y) \leq R^{u}(y), D^{l}(x) \leq D(t, x) \leq D^{u}(x) \text { for } t \geq 0, y \in \mathbb{R}_{+}^{m}, x \geq 0,
$$

and the pairs $\left(R^{u}, D^{l}\right),\left(R^{l}, D^{u}\right)$ satisfy assumptions (A2)-(A3) with $K>0$, i.e.,

(H2) $R^{l}\left(y_{1}, \ldots, y_{m}\right), R^{u}\left(y_{1}, \ldots, y_{m}\right)$ are nondecreasing functions on each $y_{k} \in[0, \infty), k=1, \ldots, m$;

(H3) there exist $K^{l}, K^{u}>0$ such that

$$
\begin{aligned}
& \left(x-K^{l}\right)\left(R^{l}(x, \ldots, x)-D^{u}(x)\right)<0 \quad \text { for } \quad x>0, x \neq K^{l}, \\
& \left(x-K^{u}\right)\left(R^{u}(x, \ldots, x)-D^{l}(x)\right)<0 \quad \text { for } \quad x>0, x \neq K^{u} .
\end{aligned}
$$

Then (3.1) is permanent (in $C_{0}$ ); to be more precise, all positive solutions of (3.1) satisfy

$$
K^{l} \leq \liminf _{t \rightarrow \infty} x(t) \leq \limsup _{t \rightarrow \infty} x(t) \leq K^{u} .
$$

Proof. As for the first part of the proof of Theorem 1.1. we compare the solutions $x(t)=x(t ; \varphi)(\varphi \in$ $C_{0}$ ) of equation (3.1) with the solutions $u(t)=u(t ; \varphi)$ and $v(t)=v(t ; \varphi)$ of the cooperative equations

$$
\begin{aligned}
& \dot{u}(t)=R^{u}\left(u\left(t-\tau_{1}(t)\right), \ldots, u\left(t-\tau_{m}(t)\right)\right)-D^{l}(u(t)), \\
& \dot{v}(t)=R^{l}\left(v\left(t-\tau_{1}(t)\right), \ldots, v\left(t-\tau_{m}(t)\right)\right)-D^{u}(v(t)), \quad t \geq 0,
\end{aligned}
$$

respectively. By Theorem 5.1.1 in [7] we deduce that $v(t) \leq x(t) \leq u(t)$ for $t \geq 0$, whereas Theorem 2.1 applied to these equations implies that $v(t) \rightarrow K^{l}, u(t) \rightarrow K^{u}$ as $t \rightarrow \infty$. This implies (3.2).

Remark 3.1. Of course, if in (H1)-(H3) only the conditions regarding $R^{l}$ and $D^{u}$ are assumed (and with no constraints on upper bounds for $R(t, y)$ and lower bounds for $D(t, x)$ ), instead of the permanence, only the uniform persistence for (3.1) is derived. Similarly, if only the conditions regarding $R^{u}$ and $D^{l}$ are assumed, instead of the permanence, one simply gets that (3.1) is dissipative. On the other hand, it is apparent that more general models with distributed delays can be considered, in which case Theorem 2.2 should be used for results of comparison with auxiliary cooperative systems. 
The same technique and Corollary 2.1 lead to the corollary below.

Corollary 3.1. Let $m \in \mathbb{N}, r_{k}(t, y), d(t, x), \tau_{k}(t)$ be continuous with $0 \leq \tau_{k}(t) \leq \tau$, for $t, x \geq 0,1 \leq$ $k \leq m$, and assume that:

(i) there are (locally Lipschitz) continuous functions $r_{k}^{l}, r_{k}^{u}: \mathbb{R}_{+} \rightarrow \mathbb{R}_{+}, d^{l}, d^{u}: \mathbb{R}_{+} \rightarrow \mathbb{R}_{+}$such that

$$
r_{k}^{l}(x) \leq r_{k}(t, x) \leq r_{k}^{u}(x), d^{l}(x) \leq d(t, x) \leq d^{u}(x) \quad \text { for } \quad t \geq 0, x \geq 0 ;
$$

(ii) $x r_{k}^{l}(x)$ and $x r_{k}^{u}(x)$ are nondecreasing functions on $[0, \infty), 1 \leq k \leq m$;

(iii) the functions $r^{u}(x)-d^{l}(x)$ and $r^{l}(x)-d^{u}(x)$, where $r^{u}(x)=\sum_{k=1}^{m} r_{k}(x), r^{l}(x)=\sum_{k=1}^{m} r_{k}^{l}(x)$, are (strictly) decreasing on $[0, \infty)$;

(iv) $r^{l}(0)-d^{u}(0)>0$ and $\lim _{t \rightarrow \infty}\left(r^{u}(x)-d^{l}(x)\right)<0$.

Then, the equation

$$
\dot{x}(t)=\sum_{k=1}^{m} x\left(t-\tau_{k}(t)\right) r_{k}\left(t, x\left(t-\tau_{k}(t)\right)\right)-x(t) d(t, x(t)), \quad t \geq 0,
$$

is permanent in $C_{0}$. Moreover, (3.2) holds with $K^{u}, K^{l}$ the positive solutions of the equations $r^{u}(x)-$ $d^{l}(x)=0, r^{l}(x)-d^{u}(x)=0$, respectively.

We finally study the permanence of (1.8), with less constraints than the ones proposed in [3].

Theorem 3.2. Consider the equation

$$
\dot{x}(t)=\sum_{k=1}^{m} \frac{\alpha_{k}(t) x\left(t-\tau_{k}(t)\right)}{1+\beta_{k}(t) x\left(t-\tau_{k}(t)\right)}-\mu(t) x(t)-\kappa(t) x^{2}(t), \quad t \geq 0,
$$

where $\alpha_{k}, \kappa:[0, \infty) \rightarrow(0, \infty)$ are continuous, bounded below and above by positive constants, and $\mu, \beta_{k}, \tau_{k}:[0, \infty) \rightarrow[0, \infty)$ are continuous and bounded, for $1 \leq k \leq m$. If

$$
\sum_{k=1}^{m} \inf _{t \geq 0} \alpha_{k}(t)>\sup _{t \geq 0} \mu(t)
$$

then all solutions $x(t)=x(t ; \varphi)\left(\varphi \in C_{0}\right)$ of (3.3) satisfy the uniform estimates

$$
m_{0} \leq \liminf _{t \rightarrow \infty} x(t) \leq \limsup _{t \rightarrow \infty} x(t) \leq M_{0},
$$

where

$$
M_{0}=\limsup _{t \rightarrow \infty} \frac{1}{\kappa(t)}\left(\sum_{k=1}^{m} \alpha_{k}(t)-\mu(t)\right)
$$

and

$$
m_{0}=\frac{c_{0}}{c_{1}} \quad \text { for } \quad c_{0}=\sum_{k=1}^{m} \inf _{t \geq 0} \alpha_{k}(t)-\sup _{t \geq 0} \mu(t), c_{1}=\sup _{t \geq 0} \kappa(t)+\sum_{k=1}^{m} \inf _{t \geq 0} \alpha_{k}(t) \sup _{t \geq 0} \beta_{k}(t),
$$

For the particular case of (3.3) with $\beta_{k} \equiv 0,1 \leq k \leq m$, i.e.,

$$
\dot{x}(t)=\sum_{k=1}^{m} \alpha_{k}(t) x\left(t-\tau_{k}(t)\right)-\mu(t) x(t)-\kappa(t) x^{2}(t), \quad t \geq 0,
$$

where $\alpha_{k}, \mu, \kappa$ and $\tau_{k}$ are as above, the lower bound in (3.5) can be taken as

$$
m_{0}=\liminf _{t \rightarrow \infty} \frac{1}{\kappa(t)}\left(\sum_{k=1}^{m} \alpha_{k}(t)-\mu(t)\right) .
$$


Proof. In what follows, we set $0 \leq \tau_{k}(t) \leq \tau$ for $t \geq 0, k=1, \ldots, m$, and use the notations

$$
\underline{f}=\inf _{t \geq 0} f(t), \quad \bar{f}=\sup _{t \geq 0} f(t),
$$

for $f$ replaced by $\alpha_{k}, \beta_{k}, \mu$ or $\kappa$. By assumption, the functions $\alpha_{k}, \kappa$ are bounded and bounded away from zero, and $\beta_{k}, \mu$ are positive and bounded. Note that the cases $\underline{\mu}=0$ or $\underline{\beta_{k}}=0$, for all or some $k$ 's, are included in our setting. For $R_{k}(t, x):=\frac{\alpha_{k}(t) x}{1+\beta_{k}(t) x}$, we have $\frac{\partial}{\partial x} \overline{R_{k}}(t, x)>\overline{0}$ for $t, x \geq 0,1 \leq k \leq m$; in particular (3.3) satisfies the quasimonotone condition. Next, denote

$$
r^{u}(x)=\sum_{k=1}^{m} r_{k}^{u}(x), r^{l}(x)=\sum_{k=1}^{m} r_{k}^{l}(x), \quad D^{u}(x)=x d^{u}(x), D^{l}(x)=x d^{l}(x)
$$

where

$$
r_{k}^{u}(x)=\frac{\bar{\alpha}_{k}}{1+\underline{\beta_{k}} x}, \quad r_{k}^{l}(x)=\frac{\underline{\alpha}_{k}}{1+\overline{\beta_{k}} x} \quad \text { for } \quad x \geq 0, k=1, \ldots, m,
$$

and $d^{u}(x)=\bar{\mu}+\bar{k} x, d^{l}(x)=\underline{\mu}+\underline{k} x$ for $x \geq 0$.

The functions $x r_{k}^{u}(x), x r_{k}^{l}(\bar{x})$ are increasing and $r^{u}(x)-d^{l}(x), r^{l}(x)-d^{u}(x)$ are decreasing on $\mathbb{R}_{+}$. On the other hand, $r^{u}(\infty)-d^{l}(\infty)=-\infty$ and (3.4) implies that $r^{l}(0)-d^{u}(0)>0$. By Corollary 3.1. (3.3) is permanent; furthermore, solutions $x(t)$ of (3.3) with initial conditions in $C_{0}$ satisfy $K^{l} \leq \underline{x} \leq \bar{x} \leq K^{u}$, for $\underline{x}:=\liminf _{t \rightarrow \infty} x(t), \bar{x}:=\limsup _{t \rightarrow \infty} x(t)$, where $K^{l}, K^{u}$ are the globally attractive positive equilibria of

$$
\begin{aligned}
& \dot{u}(t)=\sum_{k=1}^{m} u\left(t-\tau_{k}(t)\right) r_{k}^{u}\left(u\left(t-\tau_{k}(t)\right)\right)-u(t) d^{l}(u(t)), \\
& \dot{v}(t)=\sum_{k=1}^{m} v\left(t-\tau_{k}(t)\right) r_{k}^{l}\left(v\left(t-\tau_{k}(t)\right)\right)-v(t) d^{u}(v(t)),
\end{aligned}
$$

respectively.

To prove the uniform upper bound in (3.5)-(3.6), we reason along the lines of the proof of Theorem 1.1. so some details are omitted. Take a sequence $\left(t_{n}\right)$ with $t_{n} \rightarrow \infty, \dot{x}\left(t_{n}\right) \rightarrow 0$ and $x\left(t_{n}\right) \rightarrow \bar{x}$. For any $\varepsilon>0$ small and $n$ large, we derive

$$
\dot{x}\left(t_{n}\right) \leq k\left(t_{n}\right)\left[\frac{1}{k\left(t_{n}\right)}\left(\sum_{k=1}^{m} R_{k}\left(t_{n}, \bar{x}+\varepsilon\right)-\mu\left(t_{n}\right) x\left(t_{n}\right)\right)-x^{2}\left(t_{n}\right)\right] .
$$

Taking limits $n \rightarrow \infty, \varepsilon \rightarrow 0^{+}$, this inequality yields

$$
\bar{x} \leq \limsup _{t \rightarrow \infty} \frac{1}{k(t)}\left(\sum_{k=1}^{m} \frac{\alpha_{k}(t)}{1+\beta_{k}(t) \bar{x}}-\mu(t)\right) \leq M_{0}
$$

For the lower bound $m_{0}$ in (3.7), we note that $K^{l}$ is the unique $x>0$ such that $r^{l}(x)-d^{u}(x)=0$. Since $r^{l}(0)-d^{u}(0)=c_{0}$ and $\left|\left(r^{l}-d^{u}\right)^{\prime}(x)\right| \leq \sum_{k=1}^{m} \underline{\alpha}_{k} \bar{\beta}_{k}+\bar{\kappa}=c_{1}$, by the Mean Value theorem we get $K^{l} \geq c_{0} / c_{1}$. In the case of (3.8), we now take a sequence $\left(s_{n}\right)$ with $s_{n} \rightarrow \infty, \dot{x}\left(s_{n}\right) \rightarrow 0$ and $x\left(s_{n}\right) \rightarrow \underline{x}$. For any $\varepsilon>0$ small and $n$ large, we derive

$$
\dot{x}\left(s_{n}\right) \geq k\left(t_{n}\right)\left[\frac{1}{k\left(s_{n}\right)}\left((\underline{x}-\varepsilon) \sum_{k=1}^{m} \alpha_{k}\left(s_{n}\right)-\mu\left(s_{n}\right) x\left(s_{n}\right)\right)-x^{2}\left(s_{n}\right)\right] .
$$

Taking limits $n \rightarrow \infty, \varepsilon \rightarrow 0^{+}$, this leads to $\underline{x} \geq m_{0}$ for $m_{0}$ as in (3.9). 
Remark 3.2. For eq. (3.8) with $\mu \equiv 0$, we obtain (1.1). Thus, Theorem 1.1 is simply a corollary of Theorems 3.2, the latter one under slightly weaker hypotheses.

Clearly, the method presented in this note applies to other scalar nonautonomous delayed population models, as illustrated in the next example.

Consider the scalar DDE

$$
\dot{x}(t)=-d(t) x(t)+\sum_{k=1}^{m} \beta_{k}(t) x\left(t-\tau_{k}(t)\right) e^{-x\left(t-\tau_{k}(t)\right)},
$$

where $\beta_{k}, \tau_{k}, d$ are continuous, bounded and nonnegative on $[0, \infty)$. Eq. (3.10) is a generalization of the well-known Nicholson's equation $\dot{x}(t)=-d x(t)+\beta x(t-\tau) e^{-x(t-\tau)}(d, \beta, \tau>0)$. The autonomous version of (3.10) reads as

$$
\dot{x}(t)=-d x(t)+\sum_{k=1}^{m} \beta_{k} x\left(t-\tau_{k}\right) e^{-x\left(t-\tau_{k}\right)} .
$$

With $d>0, \beta_{k} \geq 0$ and $\beta:=\sum_{k=1}^{m} \beta_{k}>0$, Liz et al. [6] proved that if $1<\beta / d \leq e^{2}$, then the positive equilibrium $x^{*}:=\log (\beta / d)$ of (3.11) is a global attractor of all positive solutions. Although (3.11) is not cooperative, if $1<\beta / d \leq e$ then all solutions satisfy $\lim _{t \rightarrow \infty} x(t)=x^{*} \leq 1$, so (3.11) has a cooperative large-time behavior, since $h(x):=x e^{-x}$ is increasing on $[0,1]$. Under some further constraints on the coefficients $\beta_{k}(t), d(t)$, we shall take advantage of this monotonicity to study the permanence of (3.10).

Theorem 3.3. For (3.10), suppose that

$$
\sup _{t \geq 0} d(t)<\sum_{k=1}^{m} \inf _{t \geq 0} \beta_{k}(t), \quad \sum_{k=1}^{m} \sup _{t \geq 0} \beta_{k}(t)<e \inf _{t \geq 0} d(t) .
$$

Then, all positive solutions $x(t)$ of (3.10) satisfy

$$
\liminf _{t \rightarrow \infty} \log \left(\frac{1}{d(t)} \sum_{k=1}^{m} \beta_{k}(t)\right) \leq \liminf _{t \rightarrow \infty} x(t), \limsup _{t \rightarrow \infty} x(t) \leq \limsup _{t \rightarrow \infty} \log \left(\frac{1}{d(t)} \sum_{k=1}^{m} \beta_{k}(t)\right) .
$$

Proof. With the notation $\underline{\beta}_{k}=\inf _{t \geq 0} \beta_{k}(t), \bar{\beta}_{k}=\sup _{t \geq 0} \beta_{k}(t), \underline{d}=\inf _{t \geq 0} d(t), \bar{d}=\sup _{t \geq 0} d(t)$, conditions (3.12) are written as $\bar{d}<\sum_{k=1}^{m} \underline{\beta}_{k} \leq \sum_{k=1}^{m} \bar{\beta}_{k}<e \underline{d}$. Solutions of (3.10) satisfy

$$
-\bar{d} x(t)+\sum_{k=1}^{m} \underline{\beta}_{k} h\left(x\left(t-\tau_{k}(t)\right)\right) \leq \dot{x}(t) \leq-\underline{d} x(t)+\sum_{k=1}^{m} \bar{\beta}_{k} h\left(x\left(t-\tau_{k}(t)\right)\right),
$$

where $h(x)=x e^{-x}$ for $x \geq 0$. Note also that $h(x) \leq H(x)$, where $H$ is defined by $H(x)=h(x)$ for $0 \leq x \leq 1, H(x)=e^{-1}$ for $x>1$, and that the equation $\dot{u}(t)=-\underline{d} x(t)+\sum_{k=1}^{m} \bar{\beta}_{k} H\left(x\left(t-\tau_{k}(t)\right)\right)$ is cooperative. From Corollary 3.1 (3.10) is permanent, and (3.2) holds with $m_{0}=\log \left[\left(\sum_{k=1}^{m} \underline{\beta}_{k}\right) / \bar{d}\right]>$ 0 and $M_{0}=\log \left[\left(\sum_{k=1}^{m} \bar{\beta}_{k}\right) / \underline{d}\right]<1$.

Next, the better estimates in (3.13) are derived by using the technique in the proofs of Theorems

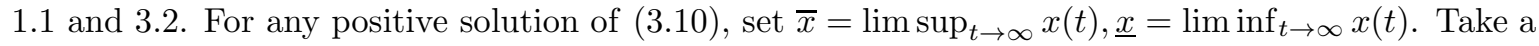
sequence $t_{n} \rightarrow \infty$ with $\dot{x}\left(t_{n}\right) \rightarrow 0, x\left(t_{n}\right) \rightarrow \bar{x}$. Fix $\varepsilon>0$. For $n$ large,

$$
\dot{x}\left(t_{n}\right) \leq-d\left(t_{n}\right) x\left(t_{n}\right)+h(\bar{x}+\varepsilon) \sum_{k=1}^{m} \beta_{k}(t)=d\left(t_{n}\right) \bar{x}\left[-1+\frac{e^{-\bar{x}}}{d\left(t_{n}\right)} \sum_{k=1}^{m} \beta_{k}\left(t_{n}\right)\right]+O(\varepsilon) .
$$

Taking $n \rightarrow \infty$ and $\varepsilon \rightarrow 0$, this yields $\bar{x} \leq \lim \sup _{t \rightarrow \infty} \log \left(\frac{1}{d(t)} \sum_{k=1}^{m} \beta_{k}(t)\right)$. A similar argument leads to $\underline{x} \geq \liminf _{t \rightarrow \infty} \log \left(\frac{1}{d(t)} \sum_{k=1}^{m} \beta_{k}(t)\right)$. 
This example also shows an obvious limitation of our method: since it relies on comparative results with cooperative equations, it cannot be invoked to deal with (3.10) in the case of the upper bound in (3.12) given by $\sum_{k=1}^{m} \bar{\beta}_{k}<e^{2} \underline{d}$. On the contrary, an advantage of the method is that it can be easily extended to deal with $n$-dimensional cooperative DDEs.

\section{Appendix}

For the sake of completeness, we include here the proof of Theorem 2.1, since the arguments in [1], based on the theory of cooperative autonomous DDEs, do not apply directly to equations of the form (2.1) due to the presence of time-varying delays. See [4, 5] for related results.

Proof of Theorem [2.1. Eq. (2.1) has the form (1.4) with $t_{0}=0$ and

$$
f(t, \varphi)=\rho(t)\left[R\left(\varphi\left(-\tau_{1}(t)\right), \ldots, \varphi\left(-\tau_{m}(t)\right)\right)-D(\varphi(0))\right] .
$$

We now consider separately the cases $K=0$ and $K>0$ in (A3).

(i) If $K=0$, we set $S=C_{0}$ in Lemma 2.1. Take $t \geq 0$ and $\varphi \in C$ with $0 \leq \varphi(\theta)<\varphi(0)$ for $\theta \in[-\tau, 0)$. From (A2), (A3), $f(t, \varphi) \leq \rho(t)[R(\varphi(0), \ldots, \varphi(0))-D(\varphi(0))]<0$. From Lemma 2.1] we deduce that all solutions $x(t)=x(t ; \varphi)$ of (2.1) are defined and bounded on $[0, \infty)$, and satisfy $0 \leq x(t) \leq\|\varphi\|_{\infty}, t \geq 0$. In particular, $x=0$ is a stable equilibrium. To prove that 0 is a global attractor, we need to prove that $\bar{x}:=\limsup _{t \rightarrow \infty} x(t)=0$ for any nonnegative solution $x(t)$.

Arguing by contradiction, we suppose that $\bar{x}>0$, and take a sequence $t_{n} \rightarrow \infty$ such that $\dot{x}\left(t_{n}\right) \rightarrow 0$ and $x\left(t_{n}\right) \rightarrow \bar{x}$ as $n \rightarrow \infty$. In order to simplify the notation, denote $R_{0}(x):=R(x, \ldots, x), x \geq 0$. Fix $\varepsilon>0$. For $n$ sufficiently large, we have $x\left(t_{n}+\theta\right) \leq \bar{x}+\varepsilon, 0 \leq \theta \leq 0$, thus

$$
\dot{x}\left(t_{n}\right) \leq \rho\left(t_{n}\right)\left[R_{0}(\bar{x}+\varepsilon)-D\left(x\left(t_{n}\right)\right)\right]
$$

Since $\rho\left(t_{n}\right) \geq \rho_{0}>0, \lim _{n} \dot{x}\left(t_{n}\right)=0, \lim _{n} D\left(\left(x\left(t_{n}\right)\right)=D(\bar{x})\right.$ and

$$
R_{0}(\bar{x}+\varepsilon)-D(\bar{x}) \rightarrow R_{0}(\bar{x})-D(\bar{x})<0 \quad \text { as } \quad \varepsilon \rightarrow 0^{+}
$$

this leads to $0 \leq \rho_{0}\left[R_{0}(\bar{x})-D(\bar{x})\right]<0$, which is a contradiction. Hence $\bar{x}=0$.

(ii) If $K>0$, we effect the change of variables $y(t)=\frac{x(t)}{K}-1$, which transforms (2.1) into

$$
\dot{y}(t)=\frac{\rho(t)}{K}\left[R\left(K+K y\left(t-\tau_{1}(t)\right), \ldots, K+K y\left(t-\tau_{m}(t)\right)\right)-D(K+K y(t))\right], t \geq 0
$$

for which $S=\{\varphi \in C: \varphi(\theta) \geq-1$ for $\theta \in[-\tau, 0), \varphi(0)>-1\}$ is the set of admissible initial conditions. Using again (2.2) and the fact that $R$ is nondecreasing on each variable, one sees that (A.1) satisfies condition (h2). Thus, Lemma 2.1 implies that all solutions $x(t)=x(t ; \varphi)$ of (2.1) are global and bounded, and that the equilibrium $x=K$ is stable.

For solutions $y(t)=y(t ; \varphi)$ of (A.1) (with $\varphi \in S)$, define now $-v=\liminf _{t \rightarrow \infty} y(t), u=$ $\lim \sup _{t \rightarrow \infty} y(t)$. We have $-1 \leq-v \leq u<\infty$. It suffices to show that $\max (u, v)=0$.

Case 1: If $\max (u, v)=u>0$, we take a sequence $t_{n} \rightarrow \infty$ such that $\dot{y}\left(t_{n}\right) \rightarrow 0$ and $y\left(t_{n}\right) \rightarrow u$ as $n \rightarrow \infty$. Reasoning as above, for any $\varepsilon>0$ small and $n$ large, we obtain

$$
\dot{y}\left(t_{n}\right) \leq \frac{\rho\left(t_{n}\right)}{K}\left[R_{0}(K(1+u+\varepsilon))-D\left(K\left(1+y\left(t_{n}\right)\right)\right)\right]
$$

with $\rho\left(t_{n}\right) \geq \rho_{0}>0$, and $R_{0}(K(1+u+\varepsilon))-D\left(K\left(1+y\left(t_{n}\right)\right)\right) \rightarrow R_{0}(K(1+u))-D(K(1+u))$ as $\varepsilon \rightarrow 0^{+}$. Hence $0 \leq R_{0}(K(1+u))-D(K(1+u))$, which is not possible with $u>0$. 
Case 2: If $\max (u, v)=v>0$, from the above case we may consider $u<v$. We first prove that $v<1$. Choose $\varepsilon>0$ small so that $u+\varepsilon<v$, and $t_{0}$ such that $y(t) \leq u+\varepsilon$ for $t \geq t_{0}+\tau$. By Lemma 2.1 the solution $y(t)$ satisfies

$$
|y(t)| \leq \max _{s \in\left[t_{0}-\tau, t_{0}\right]}|y(s)| \leq \max \left\{u+\varepsilon, \max _{s \in\left[t_{0}-\tau, t_{0}\right]}(-y(s))\right\}=: \ell, \quad t \geq t_{0} .
$$

In particular, $y(t) \geq-\ell>-1$ for $t \geq t_{0}$, thus $-v>-1$. We now choose a sequence $s_{n} \rightarrow \infty$ with $\dot{y}\left(s_{n}\right) \rightarrow 0$ and $y\left(s_{n}\right) \rightarrow-v$ as $n \rightarrow \infty$. Proceeding along the lines of the above case, we now get $0 \geq R_{0}(K(1-v))-D(K(1-v))$, which contradicts (2.2). The proof is complete.

\section{Acknowledgement}

The research was supported by Fundação para a Ciência e a Tecnologia (Portugal), PEst-OE/MAT/UI0209/2011.

\section{References}

[1] J. Arino, Lin Wang and Gail S.K. Wolkowicz, An alternative formulation for a delayed logistic equation, J. Theor. Biol. 241 (2006), 109-119.

[2] L. Berezansky, J. Bastinec, J. Diblik and Z. Smarda, On a delay population model with quadratic nonlinearity, Adv. Difference Equ. 2012 (2012), 230.

[3] J. Bastinec, L. Berezansky, J. Diblik and Z. Smarda, On a delay population model with a quadratic nonlinearity without positive steady state, Appl. Math. Comput. 227 (2014), 622-629.

[4] T. Faria, Asymptotic stability for delayed logistic type equations, Math. Comput. Modelling 43 (2006), 433-445.

[5] Y. Kuang, Delay Differential Equations with Applications in Population Dynamics, Academic Press, London, 1992.

[6] E. Liz, M. Pinto, V. Tkachenko and S. Trofimchuk, A global stability criterion for a family of delayed population models, Quart. Appl. Math. 63 (2005), 56-70.

[7] H.L. Smith, Monotone Dynamical Systems. An Introduction to the Theory of Competitive and Cooperative Systems, Mathematical Surveys and Monographs, Amer. Math. Soc., Providence, 1995. 